\title{
The advantages of keeping your offspring quiet
}

Plants can counteract infections with plant pathogens in part by expressing resistance $(R)$ genes that target patho-

all of the

progeny, even

the F2 and F3 generations, inherited Avr3a silencing.

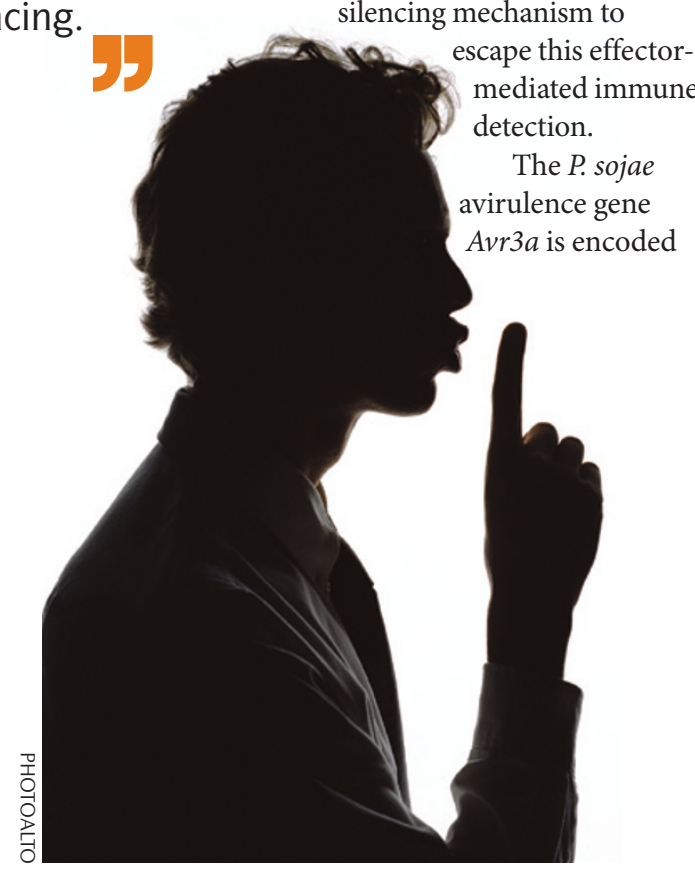

in a highly polymorphic region of the genome. Four Avr3a haplotypes have been identified, each of which expresses virulent or avirulent alleles of Avr3a. To investigate the inheritance of the different haplotypes, Gijzen and colleagues outcrossed the avirulent $P$. sojae str. P7076 $\left(A v r 3 a^{P 7076} / A v r 3 a^{P 7076}\right)$ with the virulent $P$. sojae str. ACR10 (Avr3a $a^{A C R 10} / A v r 3 a^{A C R R 10}$ ). As expected, the parental ACR10 strain caused disease in all plants, and the parental P7076 strain infected only soybeans without the corresponding $R$ gene, $R p s 3 a$. The outcross revealed an unexpected pattern that did not match the previously described dominance of avirulent Avr genes: all the $A v r 3 a^{P 7076 / A v r} 3 a^{A C R 10} \mathrm{~F}_{1}$ progeny could infect soybean plants irrespective of the presence of the host $R$ gene. According to Mendelian genetics, further crossing should produce a mixture of virulent and avirulent progeny strains; however, all $\mathrm{F}_{2}$ and $\mathrm{F}_{3}$ progeny were virulent, even those that were homozygous for the avirulent $A v r 3 a^{P 7076}$ allele.

Why were all the progeny strains able to cause disease even though some had an avirulent genotype? To answer this question, the authors looked at the mRNA levels of Avr3a. P. sojae str. ACR10 lacks Avr3a transcripts and therefore cannot be targeted by the corresponding plant resistance gene, whereas P. sojae str. P7076 expresses Avr3a mRNA, which is targeted by the host Rps3a gene. Surprisingly, all of the progeny, even the F2 and F3 generations, inherited $A v r 3 a$ silencing. Furthermore, the parental ACR10 strain and the progeny from the crosses harboured a pool of 24-26-nucleotide small RNAs (sRNAs) covering Avr3a. Previous studies have shown that such sRNAs are involved in DNA methylation and gene silencing in plants. Thus, the results suggest that $P$. sojae uses sRNA-mediated epigenetic modification of avirulence genes to evade host immune detection over multiple generations.

Transgenerational gene silencing has the potential to spread rapidly in a pathogen population, but as the gene sequence remains intact, the adaptation is potentially reversible. This gene silencing thus offers a potent and flexible mechanism of adapting to host immunity and could be present in other pathogens.

Ursula Hofer

ORIGINAL RESEARCH PAPER Qutob, D. et al. Transgenerational gene silencing causes gain of virulence in a plant pathogen. Nature Commun. 4, 1349 (2013) 